\title{
SIX2 haploinsufficiency causes conductive hearing loss with ptosis in humans
}

\author{
Jing Guan ${ }^{1,3}$, Dayong Wang ${ }^{1,3}$, Wenjian Cao ${ }^{2}$, Yali Zhao ${ }^{1}$, Renqian $\mathrm{Du}^{2}, \mathrm{Hu}$ Yuan ${ }^{1}$, Qiong Liu ${ }^{1}$, Lan Lan ${ }^{1}$, \\ Liang Zong ${ }^{1}$, Ju Yang ${ }^{1}$, Zifang Yin ${ }^{1}$, Bing Han $^{1}$, Feng Zhang ${ }^{2}$ and Qiuju Wang ${ }^{1}$
}

The ossicles represent one of the most fundamental morphological features in evolutionary biology of the mammalians.

The mobile ossicular morphology abnormalities result in the severe conductive hearing loss. Development and patterning of the middle ear malformation depend on genetic and environmental causes. However, the genetic basis for the risk of congenital ossicle malformation is poorly understood. We show here nine affected individuals in a Chinese pedigree who had bilateral conductive hearing loss with ptosis. We performed whole-genome sequencing and array comparative genomic hybridization (CGH) analysis on DNA samples from the Chinese pedigree. We confirmed the presence of a novel $60 \mathrm{~kb}$ heterozygous deletion in size, encompassing SIX2 in our family. Mutation screening in 169 sporadic cases with external ear and middle ear malformations identified no pathogenic variant or polymorphism. We suggest SIX2 haploinsufficiency as a potential congenital factor could be attributed to developmental malformation of the middle ear ossicles and upper eyelid. To the best of our knowledge, this is the first report to provide a description of copy number variation in the SIX2 gene resulting in syndromic conductive hearing loss. Journal of Human Genetics (2016) 61, 917-922; doi:10.1038/jhg.2016.86; published online 7 July 2016

\section{INTRODUCTION}

The mammalian middle ear is an air-filled cavity harboring a chain of three ossicles (malleus, incus and stapes) that transmit and amplify sound optimally from the external environment. Abnormal middle ear development leads to congenital conductive hearing loss. Malformed ossicles are the common developmental malformation of the middle ear in humans and can occur as an isolated defect or as part of syndromic defects. Gene-inactivation experiments have implicated genes required for the formation of different middle ear components. ${ }^{1}$ More recently, 40 genes have been reviewed to have a role in the formation of the middle ear. ${ }^{2}$ In particular, several homeobox genes primarily regulate the formation of specific parts of the middle ear. These include genes such as EYA1, SIX5 or SIX1 that are involved in various forms of Branchio-oto-renal syndrome (MIM \#113650, \#608389, \#610896), and HOXA1, HOXA2, $D L X 1, D L X 2$ and DLX5 that are essential for the development of pharyngeal arches. However, the list of currently known genes is still far from complete for an understanding of the formation of functional middle ear ossicles.

We show here a Chinese pedigree with conductive hearing loss with ptosis, the family members of which had a novel $60 \mathrm{~kb}$ deletion encompassing SIX2 on 2p21. To date, all reported patients with SIX2 mutation have been associated with kidney and/or urinary tract development ${ }^{3}$ the anterior cranial base development, ${ }^{4,5}$ the pyloric sphincter formation, ${ }^{6}$ and limb tendon development. ${ }^{7}$ However, we did not find any associated pedigree phenotypes noted in OMIM
(Online Mendelian Inheritance in Man), Decipher or Ensembl for SIX2. SIX2 function has not yet been characterized. Our findings may link SIX2 to human congenital ossicle malformation with ptosis disorders.

\section{MATERIALS AND METHODS}

Ethics statement

The study was approved by the Committee of Medical Ethics of Chinese People's Liberation Army (PLA) General Hospital. We obtained a written informed consent from all the participants in this study. Written informed consent was obtained from the next of kin on behalf of the minor/child participants involved in this study.

\section{Subjects}

A four-generation Chinese pedigree with 24 members segregating in autosomal dominant inheritance was investigated in the Department of Otolaryngology, Head and Neck Surgery, the Chinese PLA General Hospital, China (Figures 1a-e). A total of 14 individuals from the family were recruited subject to informed consent as approved by the committee of medical ethics of Chinese PLA General Hospital.

All participants underwent clinical, audiological and ophthalmic evaluation, including physical examination, otoscopic examination, pure-tone audiometry (EAR-3A insert earphones), tuning fork tests, tympanometry, acoustic reflex, distortion product evoked otoacoustic emissions and auditory brainstem responses using IHS Smart EP 4009 (Miami, FL, USA; Supplementary Table S1). The audiological data were evaluated based on the criteria established by European Working Group on Genetics of Hearing loss. Air conduction thresholds were bilaterally determined at octave frequencies $0.25-8.0 \mathrm{kHz}$ and

\footnotetext{
${ }^{1}$ Department of Otolaryngology-Head and Neck Surgery, Chinese PLA Institute of Otolaryngology, Chinese PLA General Hospital, Beijing, China and ${ }^{2}$ State Key Laboratory of Genetic Engineering, School of Life Sciences, Fudan University, Shanghai, China

${ }^{3}$ These authors contributed equally to this work.

Correspondence: Professor Q Wang, Department of Otolaryngology-Head and Neck Surgery, Chinese PLA Institute of Otolaryngology, 28 Fuxing Road, Beijing, 100853, China. E-mail: wqcr@263.net or wqcr301@sina.com

Received 21 January 2016; revised 26 March 2016; accepted 25 May 2016; published online 7 July 2016
} 
a

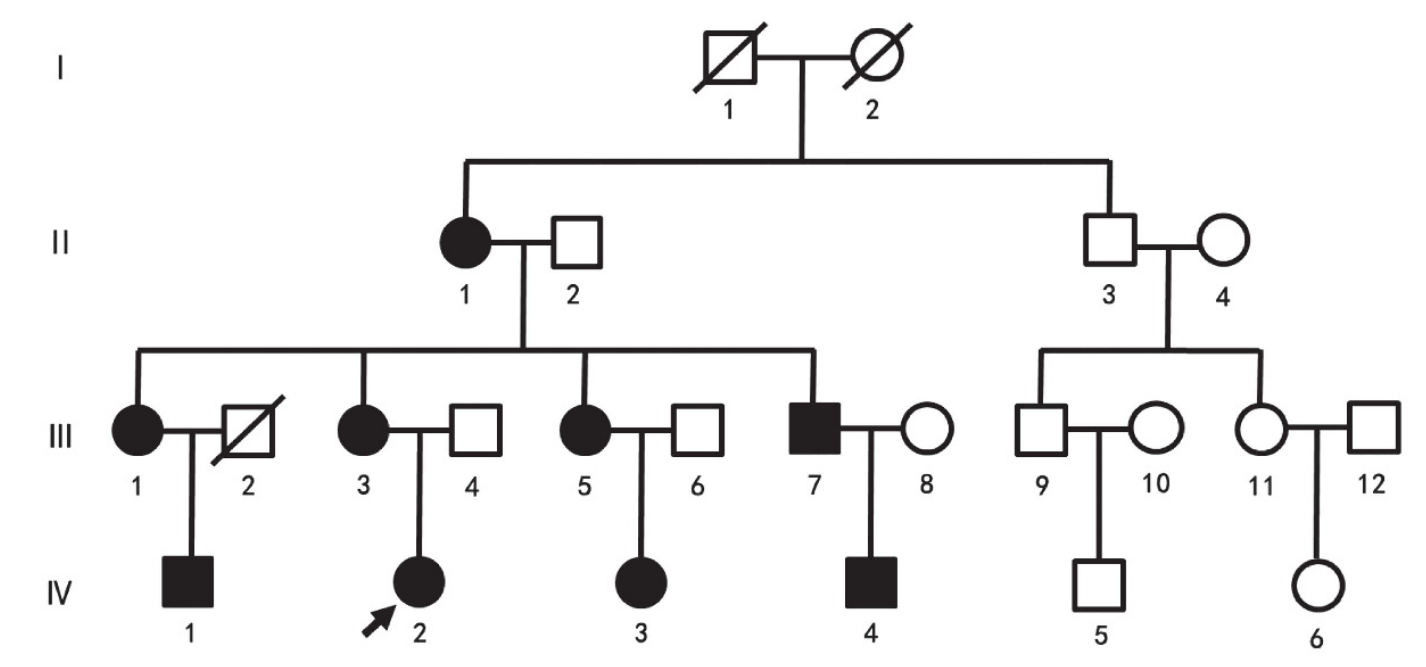

b
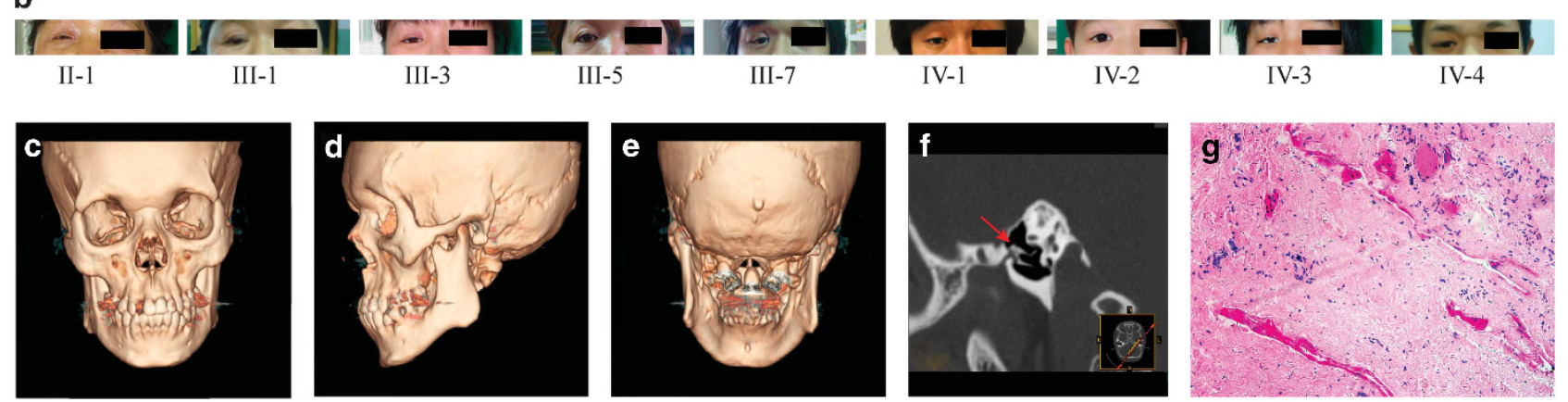

Figure 1 (a) Pedigree. Filled black symbols for males (squares) and females (circles) represent affected individuals (II-1, III-1,3,5,7, IV-1,2,3,4), and empty symbols represent unaffected ones. An arrow denotes the proband. (b) The eye photographs of patients. The affected member underwent surgical ptosis corrections. (c-e) Cranial computed tomography (CT) three-dimensional (3D) reconstruction for the patient IV-1. (f) High-resolution computed tomography (HRCT) was performed in IV-2 subject and showed abnormality of middle ear that include fusions of malleus and incus (red arrowhead). (g) Pathological examination of levator muscles of upper eyelids (IV-1) showed skeletal muscles with fibrous tissue hyperplasia and hyaline degeneration.

bone conduction thresholds at octave frequencies $0.25-4.0 \mathrm{kHz}$. Masking noise was presented at the contralateral ear through a headphone to assess masked air conduction and bone conduction thresholds according to Hood's plateau method, when interaural differences in air conduction or intra-aural air-bone gap were beyond 40 and $10 \mathrm{~dB}$, respectively. Normal hearing corresponded to air conduction thresholds of $<15 \mathrm{~dB}$ hearing level averaged over $0.5,1.0$ and $2.0 \mathrm{kHz}$ (Figure 2).

Temporal computed tomography (Figure 1f) and general anesthesia tympanotomy surgery were performed on some subjects (III-3, IV-1,2,3) to verify what middle ear and inner ear occurred. Ptosis was diagnosed and other ophthalmic diseases were excluded by professional ophthalmologist. Four bilateral ptosis patients (III-7, IV-1,2,3) were treated by levator muscle resection and frontalis muscle-fascial flap suspension procedure separately and surgical specimens were tested by using pathological methods. The skull threedimensional reconstruction computed tomography (IV-1,2), electrocardiogram (II-1, III-1,3, IV-1,2), abdominal ultrasonography (II-1,3, III-1,3,5,7, IV-3,4), chest X-ray (II-1, IV-2) and joint X-ray (III-3, IV-2,4) were also performed to exclude whether the family members had other complications (Supplementary Table S2). Blood samples were used for determination of standard hematological indices, liver and renal function, high-resolution karyotyping and DNA isolation for genetic analysis.

Furthermore, we evaluated 169 sporadic Chinese patients with congenital external and middle ear malformations with or without congenital ptosis, and 46 Chinese controls carried out tests of replication. All the participants were recruited to informed consent as approved by the committee of medical ethics of Chinese PLA General Hospital.
High-resolution assay of comparative genomic hybridization (CGH) microarray

Genomic DNAs of the subjects were extracted from peripheral blood samples. Sex-matched controls DNAs were adopted. These DNAs were fragmented using $A l u \mathrm{I}$ and RsaI enzyme digestion. DNA labeling was conducted using Agilent SureTag DNA Labeling Kit (Santa Clara, CA, USA). Different fluorescence dyes were used for DNA labeling of subjects (Cy5-dUTP) and controls (Cy3-dUTP). Each labeled subject DNA was hybridized together with the labeled control DNA onto Agilent SurePrint G3 human $1 \times 1 \mathrm{M}$ microarray for $40 \mathrm{~h}$ at $65^{\circ} \mathrm{C}$. DNA processing, microarray handling and scanning were conducted by following the Agilent oligonucleotide CGH protocol (version 6.0).

Genome-wide copy number variation (CNV) analyses The microarray scanning profiles were processed by Agilent Feature Extraction 10.7.3.1. The extracted data were analyzed and plotted by Agilent Workbench 7.0. ADM-2 was selected as statistical algorithm with the threshold of 6.0 and the Fuzzy Zero turning on. Each CNV was called by at least four consecutive probes with $\log _{2}$ Ratio (fluorescence value ratio of subject-associated Cy5 to control-associated Cy3) consistent with deletion or duplication. The CNV records archived in Database of Genomic Variants were used as references to exclude common CNVs in human populations and help identified rare $\mathrm{CNVs}$ that may cause the clinical conditions in our pedigree. 

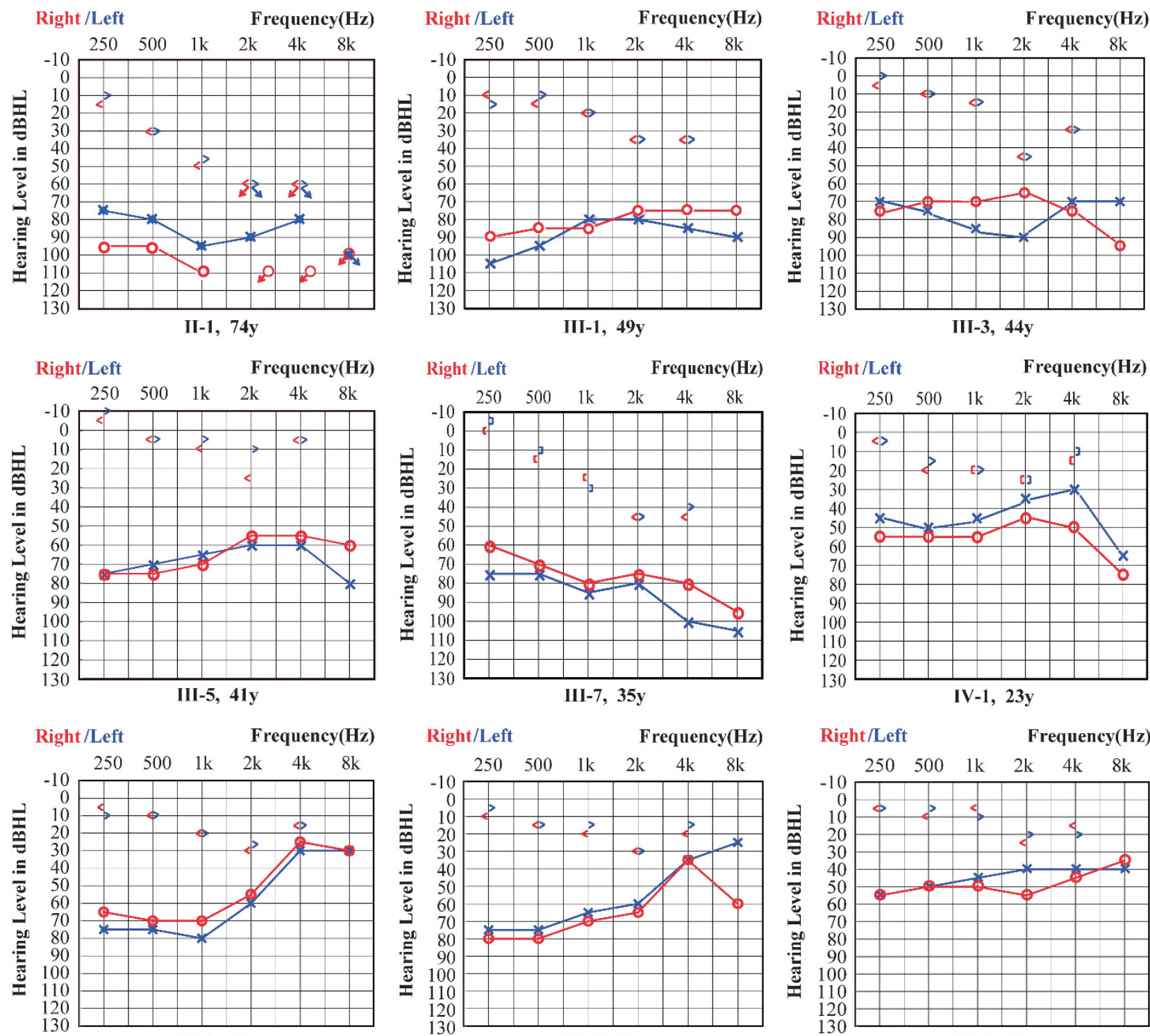

IV-2, 12y

IV-3, 18y

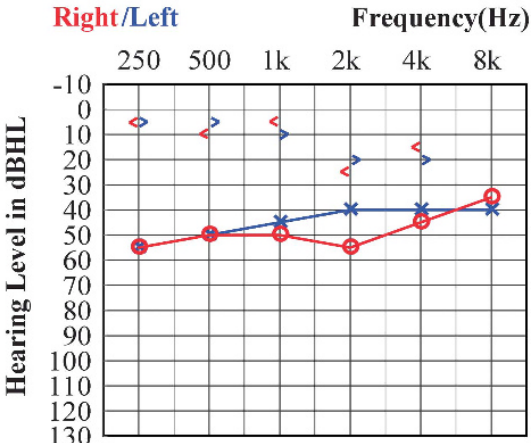

IV-4, 12y

Figure 2 Audiograms of the nine affected family members. The result of pure-tone audiometry (PTA) indicated conductive hearing loss across all frequencies in the bilateral ears. The age at the time of audiological examination was recorded. The horizontal axis shows tone frequency ( $\mathrm{Hz})$; the vertical axis gives hearing level (dB). Symbols ' 0 ' and ' $x$ ' denote air conduction pure-tone thresholds at different frequencies in the right and left ear, the symbols ' $<$ ' and ' $>$ ' denote bone conduction pure-tone thresholds at different frequencies in the right and left ear and the symbols '[' and ']' denote marked bone conduction pure-tone threshold with white noise.

\section{Whole-genome sequencing}

We performed whole-genome sequencing of two patients (IV-2 and IV-3) and one control individual (III-9) using the Illumina HiSeq 2000 platform (San Diego, CA, USA), and more than 30-fold coverage was achieved for each sample. Single-nucleotide variants were called by GATK (version 5091; http:// www.broadinstitute.org/gatk/index.php) with stringent criteria.

\section{Mutational replication test}

All the participants underwent DNA sequencing and electrophoretic condition for screening the candidate gene. PCR was performed with PE9700 thermocyclers (Applied Biosystems, Foster City, CA, USA). Sequence analysis was performed on an automated sequencer (ABI 3730, Applied Biosystems) for both affected and normal individuals. Nucleotide alterations including mutations and polymorphisms were identified by sequence alignment with the NCBI
(National Center for Biotechnology Information) Reference Sequence using the DNAStar software 5.0 versions (DNASTAR, Madison, WI, USA).

\section{RESULTS}

\section{Clinical features of this pedigree}

Based on completed medical physical examinations, all the nine affected family members (II-1, III-1,3,5,7, IV-1,2,3,4) were identified as having bilateral conductive hearing loss and congenital ptosis without facial abnormality that occurred through autosomal dominant inheritance (Figures 1 and 2). Some individuals showed elevation of bone-conduction hearing thresholds in medium-high frequency (II-1, III-1,3,7) and asymmetric audiograms (II-1, III-3, IV-1). All the affected subjects had normal auricles and bilateral stenosis without atresia of the ear canals. Four individuals (III-3, IV-1,2,3) had 
surgically confirmed congenital middle ear ossicle malformation, including fusions of malleus and incus, absent superstructure of stapes and fixation of the stapes footplate (Supplementary Table S2). Other audiological examinations, such as aural acoustic impedance, auditory brainstem response and so on, were all negative (Supplementary Table S1). Pathological examination of the levator muscles of upper eyelids (IV-1) showed skeletal muscles with fibrous tissue hyperplasia and hyaline degeneration (Figure 1g). We performed high-resolution karyotyping in one typically affected subject (III-3) and no abnormalities were found.

\section{Genome-wide CGH microarray analysis and whole-genome sequencing identified}

Each dot in Figure 3a represents a unique oligonucleotide probe corresponding to a specific locus in the human genome. The probes with increased hybridization intensity of $\log _{2}$ ratio $>0.25$ are shown in red, whereas the ones with decreased intensity of $\log _{2}$ ratio $<-0.25$ are in green. In Figure 3a (top), the CGH result showed a region in 2 p21 with continuous green dots suggesting a deletion. In Figure $3 \mathrm{a}$ (bottom), the aberration region was zoomed in and the heterozygous deletion $\left(\log _{2}\right.$ ratio $\left.\approx-1\right)$ of $\sim 60 \mathrm{~kb}$ in size caused the loss of one SIX2 gene copy. Genome-wide CGH microarray analysis identified a SIX2 gene deletion in patient IV-2 (Figure 3a).

Using whole-genome sequencing, 29 nonsynonymous coding single-nucleotide variants that were predicted to be damaging were identified as candidates, but none of them segregated with the disease phenotype in the family. Array-based CGH revealed an $\sim 60 \mathrm{~kb}$ fragment deletion that was presented in patient IV-2 (Figure 3a), and this was also confirmed by structural variation analysis based on BreakDancer (http://breakdancer.sourceforge.net/) and CNVnator (http://sv.gersteinlab.org/cnvnator/) (Figure 3b). Inspection of the Illumina reads spanning the breakpoints indicated that this 59 482-bp deletion was realized by a 12-bp microhomology between the AluSx mobile element to the left and the AluJb to the right (Figure 3c).

We specially designed three primers that included a pair encompassing the boundary of the deletion region in $2 \mathrm{p} 21$ and one primer located in the internal region. Results among the three primers, one or two bands, were amplified to identify whether or not there was heterozygous deletion. All patients lacked one copy of the SIX2 gene. Neither deletions nor duplications of this region have been involved in control individuals (Supplementary Figure S1 and Supplementary Table S3).

\section{SIX2 mutational analysis}

All the affected family members presented no pathogenic variants on the remaining allele of SIX2. We hypothesized that mutations in SIX2 could be responsible for an oriented cohort with external ear and middle ear malformations. We therefore sequenced both exons and intro-exon boundaries of SIX2 to identify possible DNA variations from 169 unrelated individuals and 46 controls. However, a

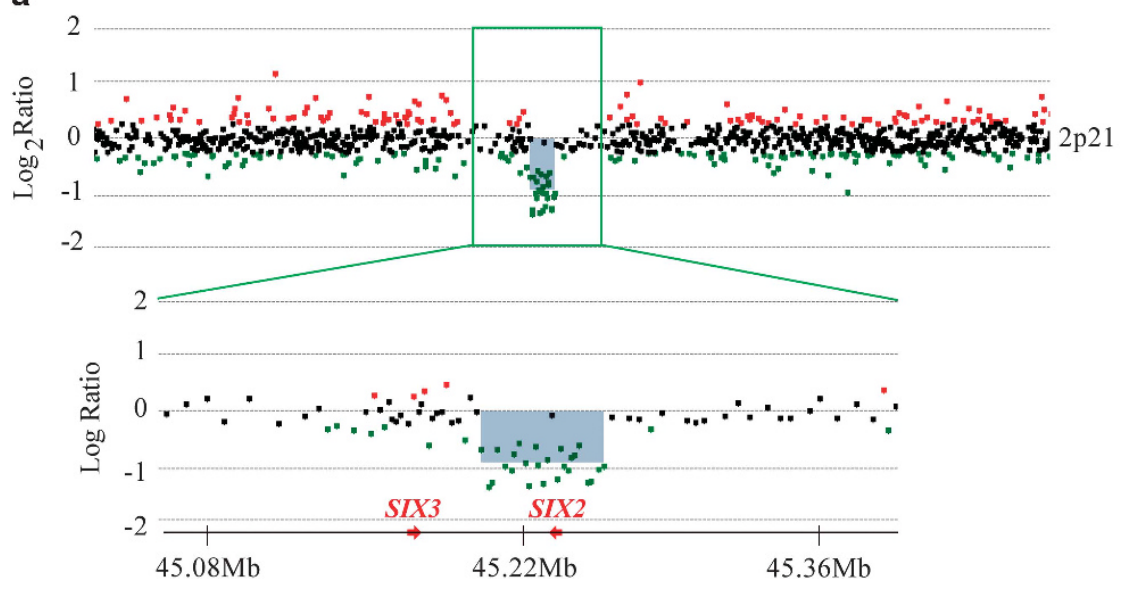

C

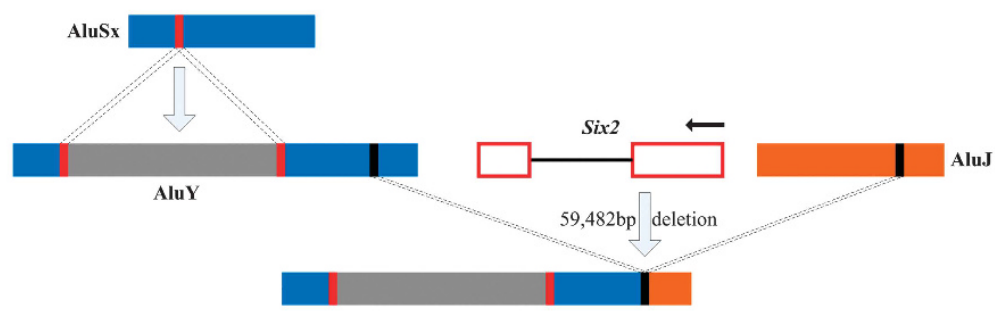

b

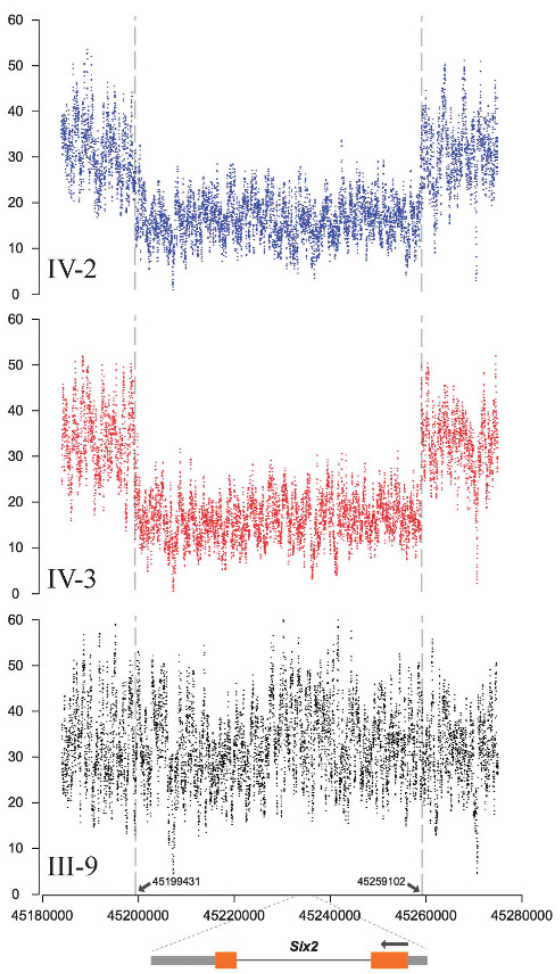

Figure 3 Genome-wide comparative genomic hybridization (CGH) microarray analysis and whole-genome sequencing identified. (a) Genome-wide CGH microarray analysis identified a SIX2 gene deletion in patient IV-2. Each dot represents a unique oligonucleotide probe corresponding to a specific locus in the human genome. The probes with increased hybridization intensity of $\log _{2}$ ratio $>0.25$ are shown in red, whereas the ones with decreased intensity of $\log _{2}$ ratio $<-0.25$ are in green. (a, top) The CGH result showed a region in 2 p21 with continuous green dots suggesting a deletion. (a, bottom) The aberration region was zoomed in and the heterozygous deletion ( $\log _{2}$ ratio $\approx-1$ ) of $\sim 60 \mathrm{~kb}$ in size caused the loss of one $S I X 2$ gene copy. (b) Whole-genome sequencing revealed an $\sim 60 \mathrm{~kb}$ fragment deletion that was present in both cases (IV-2,3) but not the control (III-9), as confirmed by structural variation analysis based on BreakDancer and CNVnator. (c) Inspection of the Illumina reads spanning the breakpoints indicated that this 59 482-bp deletion was realized by a 12-bp microhomology between the AluSx mobile element to the left and the AluJb to the right. 
no pathogenic variant or polymorphism was identified in the coding region of SIX2 in our affected subjects. We identified 1 SIX2 synonymous mutation in 10 of 169 patients and another SIX2 synonymous mutation in 1 of 46 controls that all had previously documented single-nucleotide variants (rs76298925 and rs146943650).

\section{DISCUSSION}

We show here nine affected individuals in a Chinese pedigree who had bilateral conductive hearing loss with ptosis. Moreover, the examined affected subjects did not have signs of any other abnormalities and we propose that the phenotype of the family is inconsistent with other types of craniofacial and mandibulofacial dysostosis such as Branchio-oto-renal syndrome, Treacher Collins syndrome or frontonasal dysplasia syndromes. By array CGH analysis, we confirmed the presence of a novel $60 \mathrm{~kb}$ heterozygous deletion in size, encompassing SIX2 but no other known or predicted genes, making it an excellent candidate for the phenotype in our family. Furthermore, we determined that one SIX2 gene copy is causative because they cosegregated with disease status. Generally, the molecular mechanism associated with heterozygous gene deletions is haplonsufficiency, in which half the gene dosage is not sufficient for normal function. ${ }^{8}$ SIX2 encodes a transcription factor homologous to the Drosophila homeobox sine oculis, one of the SIX family of homeobox transcription factors counting six members; members of this family are becoming recognized as a set of essential transcription factors involved in embryonic morphogenesis of cranium and renal system such as Branchio-oto-renal (BOR) syndrome caused by missense mutations in SIX1 or SIX5. It is noteworthy that in reverse transcriptase-PCR experiments, relatively strong expression was also observed in fetal limb, middle ear and kidney. ${ }^{9}$ So far, the human phenotype associated with SIX2 mutations seems restricted to kidney and urinary tract abnormalities, ${ }^{10}$ isolated microtia ${ }^{11}$ and frontonasal dysplasia syndrome. ${ }^{5}$ However, several previous reports that described patients with various abnormalities did not clearly show middle ear ossicle malformation with ptosis. Hence, we indicate a newly recognized phenotype. Moreover, for the individuals, the elevation of bone-conduction hearing thresholds in medium-high frequency or asymmetric audiograms may be associated with chronic inflammation of the middle ears.

The middle ear ossicles represent one of the most fundamental morphological features in evolutionary biology of vertebrates. ${ }^{12}$ The malleus, incus and stapes form an ossicle chain in the middle ear. The malleus and incus skeletal elements are unique to mammals. In our family, the mobile ossicular morphology abnormalities result in severe conductive hearing loss, including fusions of malleus and incus, absent superstructure of stapes and fixation of the stapes footplate. Ossicular development starts as neural crest mesenchyme from the first and second branchial arches that forms cartilaginous models of the ossicles. Except for the stapes developed from the second arch skeleton and otic capsule, ${ }^{4}$ the malleus and incus skeletal elements are derived from the first branchial arches. The incus and malleus develop from one collection of cells and then separate with formation of the malleoincudal joint by endochondral ossification.,13,14 Six2 has recently identified to be expressed predominantly in a large domain in the first branchial arch and in a restricted one in the second branchial arch. ${ }^{15}$ Six2 function is likely to target general regulators of cartilage growth and differentiation in endochondral skeleton. ${ }^{4}$ Our data suggest that SIX2 haploinsufficiency is associated with congenital ossicle malformation. Besides, ptosis is one of the most common eyelid disorders encountered in ophthalmology and can be classified as congenital or acquired. The abnormal drooping of the upper eyelid in our family reported here was present at birth. The majority of congenital ptosis is due to myogenic dysgenesis of the levator muscle. ${ }^{16}$ A study demonstrated that Six 2 gene is involved in the formation of craniofacial skeletal muscle and was transcribed in myogenic cells in the mouse embryo. Six 2 may play such a role as it is expressed at myogenic sites. ${ }^{17}$ Thus, there is a reduction or absence of functional muscle, impairing the ability of the levator to contract and elevate the eyelids. By pathological examination of levator muscles we then identified the result as skeletal muscles with fibrous tissue hyperplasia and hyaline degeneration in the family. SIX2 haploinsufficiency therefore contributes substantively to the trait of congenital ptosis.

Copy number variants are a well-recognized cause of genetic disease, typically because of changes in copy number of dosagesensitive genes, gene disruption or fusion events leading to novel genes. ${ }^{18}$ The effect of diminished SIX2 dosage in our family is complex. The formation of this mutation may be related to the chromosomal recombination induced by insertion of the Alu sequence. External ear abnormalities are often associated with middle ear abnormalities because they share a common embryologic derivative. We further sequenced SIX2 in a cohort of 169 unrelated persons with congenital external and/or middle ear malformations. However, we failed to detect any pathogenic variants. We also applied array CGH to evaluate three unrelated individuals with conductive hearing loss and ptosis but did not find the SIX2 deletion. One possibility is that the group of affect individuals was not phenotypically consistent with our family. The limited number of available affected subjects did not allow us to perform another genome-wide search. We suggest SIX2 should not be tested in patients with isolated congenital abnormalities. A second possibility is that SIX2 was of low frequency and did not completely elucidate the role in the etiology of ossicle malformation with ptosis. Development and patterning of the middle ear depend on a number of transcription factors and complex tissue interactions. ${ }^{1}$ However, the genetic basis for the risk of congenital ossicle malformation is poorly understood.

In conclusion, we propose that a Chinese pedigree represents a novel phenotype including conductive hearing loss with ptosis. We shed light on a novel $60 \mathrm{~kb}$ heterozygous deletion encompassing SIX2 on 2 p21 as a potential congenital factor that could be attributed to developmental malformation of the middle ear ossicles and upper eyelid. The comparable expression during human embryogenesis and the high protein conservation from mice to humans implicate SIX2 haploinsufficiency as potential congenital cause of conductive hearing loss with ptosis in humans. Identification of additional families with the same phenotype and knowledge about the underlying genetic pathway involved will be our next necessary step to verity this novel gene.

\section{CONFLICT OF INTEREST}

The authors declare no conflict of interest.

\section{ACKNOWLEDGEMENTS}

We thank the family for their invaluable cooperation and participation. We thank all colleagues in ENT departments who provided the patient samples. We thank Yujun Zhang for performing genome-wide CNV analyses for subjects IV-2,3 and III-9, Cui Zhao for DNA extraction and Nan Liu for SIX2 gene screening. We thank the families for their participation. This work was supported by grants of the National Key Basic Research Program of China (No. 2014CB943001), the National Natural Science Foundation of China (No. 81120108009 and 81530032) and China Postdoctoral Science Foundation (No. 2015M572766). 
1 Mallo, M. Formation of the middle ear: recent progress on the developmental and molecular mechanisms. De. Biol. 231, 410-419 (2001).

2 Chapman, S. C. Can you hear me now? Understanding vertebrate middle ear development. Front. Biosci. 16, 1675-1692 (2011).

3 Kobayashi, A., Valerius, M. T., Mugford, J. W., Carroll, T. J., Self, M., Oliver, G. et al. Six2 defines and regulates a multipotent self-renewing nephron progenitor population throughout mammalian kidney development. Cell Stem Cell 3, 169-181 (2008).

4 He, G., Tavella, S., Hanley, K. P., Self, M., Oliver, G., Grifone, R. et al. Inactivation of Six2 in mouse identifies a novel genetic mechanism controlling development and growth of the cranial base. Dev. Biol. 344, 720-730 (2010).

5 Hufnagel, R. B., Zimmerman, S. L., Krueger, L. A., Bender, P. L., Ahmed, Z. M. \& Saal, H. M. A new frontonasal dysplasia syndrome associated with deletion of the SIX2 gene. Am. J. Med. Genet. A 170A, 487-491 (2015).

6 Self, M., Geng, X. \& Oliver, G. Six2 activity is required for the formation of the mammalian pyloric sphincter. Dev. Biol. 334, 409-417 (2009).

7 Yamamoto-Shiraishi, Y. \& Kuroiwa, A. Wnt and BMP signaling cooperate with Hox in the control of Six2 expression in limb tendon precursor. Dev. Biol. 377, 363-374 (2013).

8 Cirulli, E. T. \& Goldstein, D. B. Uncovering the roles of rare variants in common disease through whole-genome sequencing. Nat. Rev. Genet. 11, 415-425 (2010).

9 Boucher, C. A., Winchester, C. L., Hamilton, G. M., Winter, A. D., Johnson, K. J. \& Bailey, M. E. Structure, mapping and expression of the human gene encoding the homeodomain protein, SIX2. Gene 247, 145-151 (2000).

10 Weber, S., Taylor, J. C., Winyard, P., Baker, K. F., Sullivan-Brown, J., Schild, R. et al SIX2 and BMP4 mutations associate with anomalous kidney development. J. Am. Soc. Nephrol. 19, 891-903 (2008).

11 Monks, D. C., Jahangir, A., Shanske, A. L., Samanich, J., Morrow, B. E. \& Babcock, M. Mutational analysis of HOXA2 and SIX2 in a Bronx population with isolated microtia. Int. J. Pediatr. Otorhinolaryngol. 74, 878-882 (2010).

12 Meng, J., Wang, Y. \& Li, C. Transitional mammalian middle ear from a new Cretaceous Jehol eutriconodont. Nature 472, 181-185 (2011).
13 Mallo, M. Embryological and genetic aspects of middle ear development. Int. J. Dev. Biol. 42, 11-22 (1998).

14 Rodriguez, K., Shah, R. K. \& Kenna, M. Anomalies of the middle and inner ear. Otolaryngol. Clin. North Am. 40, 81-96 (2007).

15 Kutejova, E., Engist, B., Self, M., Oliver, G., Kirilenko, P. \& Bobola, N. Six2 functions redundantly immediately downstream of Hoxa2. Development 135, 1463-1470 (2008).

16 Sakol, P. J., Mannor, G. \& Massaro, B. M. Congenital and acquired blepharoptosis. Curr. Opin. Ophthalmol. 10, 335-339 (1999).

17 Relaix, F., Demignon, J., Laclef, C., Pujol, J., Santolini, M., Niro, C. et al. Six homeoproteins directly activate Myod expression in the gene regulatory networks that control early myogenesis. PLoS Genet. 9, e1003425 (2013).

18 Shearer, A. E., Kolbe, D. L., Azaiez, H. Sloan, C. M. Frees, K. L., Weaver, A. E et al. Copy number variants are a common cause of non-syndromic hearing loss. Genome Med. 6, 37 (2014).

(c) (i) $(\Theta)$ This work is licensed under a Creative Commons Attribution-NonCommercial-NoDerivs 4.0 International License. The images or other third party material in this article are included in the article's Creative Commons license, unless indicated otherwise in the credit line; if the material is not included under the Creative Commons license, users will need to obtain permission from the license holder to reproduce the material. To view a copy of this license, visit http://creativecommons.org/licenses/bync-nd/4.0/

(C) The Author(s) 2016

Supplementary Information accompanies the paper on Journal of Human Genetics website (http://www.nature.com/jhg) 